\title{
The combination of computed tomography features and circulating tumor cells increases the surgical prediction of visceral pleural invasion in clinical T1NOMO lung adenocarcinoma
}

\author{
Jinghan Shi ${ }^{1 \#}$, Fei Li $^{2 \#}$, Fujun Yang ${ }^{3 \#}$, Zhengwei Dong ${ }^{4}$, Yan Jiang ${ }^{3}$, Dania Nachira ${ }^{5}$, \\ Justyna Chalubinska-Fendler ${ }^{6}$, Terence T. Sio ${ }^{7}$, Yo Kawaguchi ${ }^{8}$, Hiromitsu Takizawa ${ }^{9}$, Xiao Song ${ }^{3}$, \\ Yang $\mathrm{Hu}^{10}$, Liang Duan ${ }^{3}$
}

${ }^{1}$ Department of Endoscopy, Shanghai Pulmonary Hospital, Tongii University School of Medicine, Shanghai, China; ${ }^{2}$ Department of Radiology, Shanghai Pulmonary Hospital, Tongji University School of Medicine, Shanghai, China; ${ }^{3}$ Department of Thoracic Surgery, Shanghai Pulmonary Hospital, Tongji University School of Medicine, Shanghai, China; ${ }^{4}$ Department of Pathology, Shanghai Pulmonary Hospital, Tongji University School of Medicine, Shanghai, China; ${ }^{5}$ Department of General Thoracic Surgery, Fondazione Policlinico Universitario “A.Gemelli”, IRCCS, Rome, Italy; ${ }^{6}$ Department of Radiation Oncology, Military Institute of Medicine, Warsaw, Poland; ${ }^{7}$ Department of Radiation Oncology, Mayo Clinic, Phoenix, Arizona, USA; ${ }^{8}$ Division of General Thoracic Surgery, Department of Surgery, Shiga University of Medical Science, Shiga, Japan; ${ }^{9}$ Department of Thoracic, Endocrine Surgery and Oncology, Tokushima University Graduate School of Biomedical Sciences, Kuramotocho, Tokushima, Japan; ${ }^{10}$ Department of Respiratory and Critical Care Medicine, Shanghai Pulmonary Hospital, Tongji University School of Medicine, Shanghai, China

Contributions: (I) Conception and design: J Shi, F Li, F Yang, L Duan, Y Hu, X Song; (II) Administrative support: L Duan, Y Hu; (III) Provision of study materials or patients: Y Jiang, L Duan, Y Hu, X Song; (IV) Collection and assembly of data: J Shi, F Li, F Yang, Y Jiang; (V) Data analysis and interpretation: J Shi, F Li, F Yang, X Song; (VI) Manuscript writing: All authors; (VII) Final approval of the manuscript: All authors.

\#These authors contributed equally to this work.

Correspondence to: Liang Duan, MD; Yang Hu, PhD; Xiao Song, PhD. 507 Zhengmin Road, Shanghai Pulmonary Hospital, School of Medicine, Tongji University, Shanghai 200433, China. Email: duan-liang@163.com; huyang3141@163.com; songxiao198327@163.com.

Background: Visceral pleural invasion (VPI) is a clinical manifestation associated with a poor prognosis, and diagnosing it preoperatively is highly imperative for successful sublobar resection of these peripheral tumors. We evaluated the roles of computed tomography (CT) features and circulating tumor cells (CTCs) for improving VPI detection in patients with clinical T1N0M0 invasive lung adenocarcinoma.

Methods: Three hundred and ninety-one patients were reviewed retrospectively in this study, of which 234 presented with a pleural tag or pleural contact on CT images. CTCs positive for the foliate receptors were enriched and analyzed prior to surgery. Logistic regression analyses were performed to assess the association of CT features and CTCs with VPI, and the receiver operating characteristic (ROC) curve was generated to compare the predictive power of these variables.

Results: Patients mostly underwent either segmentectomies (18.9\%) or lobectomies (79.0\%). Only 49 of the 234 patients with pleural involvement on CT showed pathologically confirmed VPI. Multivariate logistic regression analysis revealed that CTC level $\geq 10.42 \mathrm{FU} / 3 \mathrm{~mL}$ was a significant VPI risk factor for invasive adenocarcinoma cases $\leq 30 \mathrm{~mm}$ [adjusted odds ratio $(\mathrm{OR})=4.62,95 \%$ confidence interval (CI): 2.05-10.44, $\mathrm{P}<0.001]$. Based on CT features, subgroup analyses showed that the solid portion size was a statistically significant independent predictor of VPI for these peripheral nodules with pleural tag, while the solid portion length of the interface was an independent predictor of pleural contact. The receiver operating curve analyses showed that the combination of CTC and CT features were highly predictive of VPI [area under the curve $(\mathrm{AUC})=0.921$ for pleural contact and 0.862 for the pleural tag, respectively].

Conclusions: CTC, combined with CT features of pleural tag or pleural contact, could significantly improve VPI detection in invasive lung adenocarcinomas at clinical T1N0M0 stage prior to the patient's surgery. 
Keywords: Lung adenocarcinoma; visceral pleural invasion (VPI); circulating tumor cell (CTC); computed tomography (CT); thoracic surgery

Submitted Aug 06, 2021. Accepted for publication Nov 24, 2021.

doi: $10.21037 /$ tlcr-21-896

View this article at: https://dx.doi.org/10.21037/tlcr-21-896

\section{Introduction}

Lung cancer accounts for most cancer-related deaths worldwide, and $\sim 85 \%$ of cases comprise of non-small cell lung cancer (NSCLC) (1). For operable patients, earlystage NSCLC is usually treated with surgical resection (2). Many factors can affect the postoperative prognosis of lung cancer patients, of which one of the most important features, in terms of tumoral recurrence prediction, is visceral pleural invasion (VPI) (3). VPI is defined as the tumor invading beyond the elastic layer (PL1) and being exposed to the pleural surface (PL2) (4). It has been adopted as a tumor size (T) parameter descriptor, for tumors $\leq 30 \mathrm{~mm}$, in current TNM staging system (5). Previous studies have reported that certain radiographic findings were highly predictive of VPI, including pleural contact directly with the tumor, pleural tag, and pleural retraction, as well as a higher proportion of solid tumor component and abutment of the solid portion with the pleura (6-8).

Adenocarcinoma, the most common NSCLC subtype, often occurs near peripheries of the lung, with aggressive growth and invasion (9). Furthermore, VPI is more likely to be present for this histology, especially within peripheral pulmonary nodules (10). More specifically, under the TMN staging system, T1N0M0 lung adenocarcinoma, corresponding to stage IA NSCLC adjacent to a normal lung parenchyma margin, is classified based on the following pathological characteristics: (I) tumor $\leq 3 \mathrm{~cm}$ in size; (II) no VPI; (III) main bronchus is involved, but not carina, and (IV) imaging studies depict atelectasis/obstructive pneumonitis extending to the hilum. With respect to imaging features, T1N0M0 tumors are those defined as $\leq 3 \mathrm{~cm}$ in size, being surrounded by lung parenchyma/ visceral pleura, and without any signs of invasion proximal to lobal bronchi. For this tumor type, sublobar resection is now more widely accepted as an alternative to lobectomy for stage IA NSCLC adjacent to normal lung parenchyma margins. There, wedge resections and segmentectomy have been found to yield comparable clinical outcomes, to that of lobectomy, especially for early-stage pulmonary malignancies less than $3.0 \mathrm{~cm}$ in size $(11,12)$. However, the presence of VPI is associated with a higher incidence of lymph node metastasis, making sublobar resection, with its limited lymph node sampling and dissection, a less effective treatment approach (13). As a result, survival outcomes in patients with VPI were significantly improved by lobectomy with systematic lymphadenectomy (3). Therefore, preoperative evaluation of VPI is essential to guide the potential choices of appropriate surgical procedure for early-stage peripheral lung cancer resections. The diagnostic performance of VPI based on specific pleural imaging features, including pleural attachment or tag, has an accuracy of $51.1-72.3 \%(7,8)$. This wide range poses challenges in radiographic-pathological correlation for VPI diagnoses, necessitating the use of higher-quality predictive markers, such as from liquid biopsy.

Liquid biopsy has promising applications for cancer screening and early diagnosis (14). Studies have explored the role of circulating tumor cells (CTCs) as biomarkers for lung cancer diagnosis, treatment assessment, and prediction of prognoses (15-17). CTCs are cells which have, either singly or in small clusters, detached from primary or metastatic tumors to circulate within the bloodstream. This migration from their source tumors to the bloodstream occurs through either passive infiltration or epithelial-mesenchymal transition, the latter involving the expression of various factors promoting cellular motility and basement membrane breakdown $(18,19)$. Owing to them being representative of tumor metastasis, they have become an attractive target for detecting cancer presence and progression in patients. CTCs could originate from any cancer type, such as lung, breast, colorectal, prostate and so on (20). Due to their high apoptotic rate, stemming from their exposure to myriad cell-damaging factors within the blood stream, ranging from shear stress to innate immune system proteins, CTCs are rare within the peripheral circulation, with a frequency of $<10$ cells $/ \mathrm{mL}$ blood. Therefore, efficient isolation and enrichment are essential for more accurate cancer diagnoses. Various approaches have been employed to collect and characterize 
CTCs, including CellSearch, based on immuno-magnetic binding of epithelial cell adhesion molecule (EpCAM) and cytokeratins (CK), isolation by tumor cell size (ISET) via size-dependent filtration, ligand-targeted polymerase chain reaction (LT-PCR) to detect folate receptor (FR) positive CTCs, and others $(15,21-24)$. FR is a cell surface biomarker expressed on $\sim 80 \%$ of NSCLC cells, and the LT-PCR method has demonstrated high levels of pooled sensitivity and specificity ( $76.37 \%$ sensitivity, $82.39 \%$ specificity) for FR-positive CTC, even in early tumor stages (25). This method is commonly used for collection of adenocarcinoma CTC cells. Additionally, elevated CTC counts have been reported to occur in patients with higher-stage tumors (26), emerging as a potential approach to predict the extent of tumor invasion for the future.

In the present study, we utilized a combination of 2 diagnostic methods, computed tomography (CT) scans and measuring FR-positive CTC cell counts, whose findings have previously been analyzed separately from one another, to diagnose the presence of VPI in clinical T1N0M0 lung adenocarcinoma patients. We thus investigated a cohort of lung cancer patients to determine the presence of VPI in association with CT features, such as pleural contact or pleural tags, as well as higher CTC levels. We also compared the predictive power of variables in detecting VPI for clinical T1N0M0 lung adenocarcinoma, presenting as peripheral nodules.

We present the following article in accordance with the STARD reporting checklist (available at https://dx.doi. org/10.21037/tlcr-21-896).

\section{Methods}

\section{Study population}

This retrospective study was approved by the Ethical Review Board of Shanghai Pulmonary Hospital (No. K21-012) and was conducted in accordance with the Declaration of Helsinki (as revised in 2013). Informed consents were necessarily waived due to the retrospective nature of this study. A total of 782 consecutive patients who underwent CTC detection and surgical resection for invasive adenocarcinoma, between June 2018 and January 2019, in Shanghai Pulmonary Hospital, with pathological tumor size $\leq 30 \mathrm{~mm}$, were retrospectively enrolled (CTC detection was routinely part of the standard of care for this group of patients). Exclusion criteria were as follows: (I) patients with a history of cancer or preoperative anticancer therapies, including chemotherapy, immunotherapy, and radiotherapy; (II) node-positive lung cancers as indicated by positron emission tomography (PET)/CT, or diagnosed by presurgical endobronchial ultrasound (EBUS); (III) distant tumor metastases as confirmed by clinical and radiographic findings; (IV) patients with evidence of synchronous or metachronous nodules in CT images; (V) central lung cancer; (VI) tumor size $>30 \mathrm{~mm}$ on CT scans, or (VII) lung cancer combined with pneumonia findings, and thus unable to be measured on CT images. After applying these criteria, 391 patients with T1N0M0 lung adenocarcinoma were included for analysis.

\section{Data collection, pathological analysis, and CT imaging}

Medical records, including gender, age, smoking history, surgical type, stage of TNM (8th edition), predominant histological subtype, and preoperative carcinoembryonic antigen (CEA) level, were thoroughly reviewed for all patients.

Elastic staining was used to determine the presence of VPI from paraffin-embedded tissue sections. The sections were routinely reviewed by 2 experienced pathologists and classified as PL0 (no pleural invasion beyond the elastic layer), PL1 (tumor invasion beyond the elastic layer), and PL2 (tumor invasion to the surface of the visceral pleura). PL1 and PL2 indicated the presence of VPI (27).

Chest CT scans were performed for all patients included in the study within 1 month prior to surgery, using Siemens Somatom Definition AS scanners (Siemens Medical Systems, Erlangen, Germany) or Philips Brilliance 40 scanners (Philips Healthcare, Cleveland, USA). CT parameters were $120 \mathrm{kVp}$ tube voltage, $200 \mathrm{mAs}$ tube current, pitch of $0.875-1.5$, and detector collimation of $1-2.5$. Images were reconstructed at $1.0-\mathrm{mm}$ intervals and independently reviewed by 2 experienced thoracic radiologists. Disagreements were resolved through discussions with a third radiologist with more than 20 years of clinical experience in thoracic imaging. CT characteristics were examined in both the lung (window width 1,500 $\mathrm{HU}$ and level $600 \mathrm{HU}$ ) and mediastinal (window width $350 \mathrm{HU}$ and level $50 \mathrm{HU}$ ) window settings. Records of radiographic parameters, including tumor size and location, nodule type, and solid portion size, were obtained for each patient. For tumors with pleural involvement confirmed threedimensionally on CT images, tumor-pleura relationships were classified as pleural contact (abutting the pleural surface) or pleural tag (Figure 1). A pleural tag was 


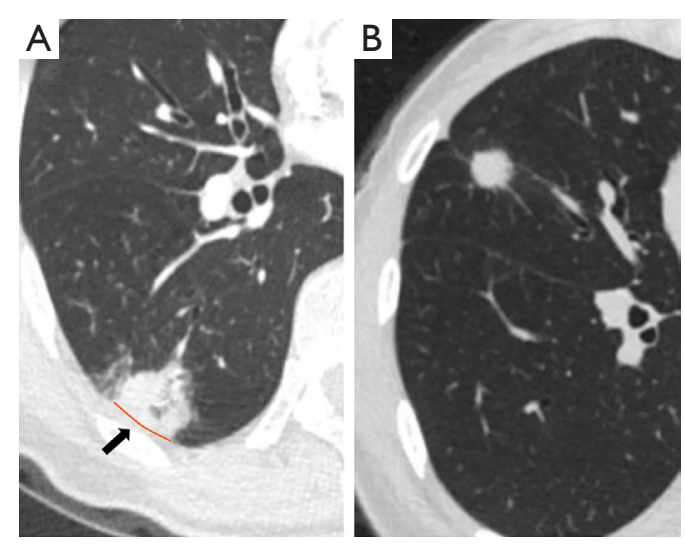

Figure 1 CT images depicting pleural contact with ISP in lung window settings (window width, 1,500 HU; window level, $-600 \mathrm{HU}$; black arrow, length of the solid portion between tumor and pleura) (A), and pleural tag, represented by linear pleural tag with soft tissue component in lung window settings (B). CT, computed tomography; ISP, interface solid portion.

characterized as one or more linear soft tissue strands, appearing as continuous linear opacities on CT scans, extending from the tumor surface to contact perpendicularly with the pleural surface. These tags were likely the result of the thickening of the interlobular septa of the lung, possibly due to pathological processes associated with tumor development, including fibrosis (28-30). The interface solid portion (ISP) length was measured as the solid portion length of tumor-pleura interface. The proportion of the length of the involved interface was calculated as: (tumorpleura interface length)/(maximum tumor diameter) $\times 100 \%$. Minimum distances between nodules and pleura were measured in nodules with a pleural tag.

\section{CTC detection}

Three milliliters of peripheral venous blood were collected alongside routine preoperative laboratory tests from each patient within 3 days before surgery. Whole blood samples were kept in ethylenediaminetetraacetic acid (EDTA) tubes at $4{ }^{\circ} \mathrm{C}$ and processed on the same day. A CTC detection kit (GenoSaber Biotech, Shanghai, China) was used for CTC analysis, as previously reported (16). Briefly, after erythrocyte lysis, CTCs were isolated using anti-CD45/CD14 immuno-magnetic beads to deplete leukocytes, and specific detection probes were added to label FRpositive CTCs, which were then subjected to quantitative PCR analysis. A diagnostic threshold of 8.7 functional units (FU) per $3 \mathrm{~mL}$ of blood was used to distinguish lung malignancies from benign disease processes.

\section{Statistical analysis}

Continuous variables were presented as median with interquartile range (IQR), and Mann-Whitney $U$ test was performed for two-group comparisons with or without VPI. For categorical variables, differences in clinicopathological characteristics between patients, with or without VPI, were evaluated using Pearson's Chi-square $\left(\chi^{2}\right.$ test) or Fisher's exact tests. Univariate logistic regression analysis was used to evaluate the association between clinicopathological characteristics and VPI. All variables that were found to be significant in the univariate analysis were included in the multivariate logistic regression model to further assess the independent risk factors associated with VPI. Tumor size was divided into 3 groups, based on their TNM stage, as categorical variables for logistic regression analysis. The receiver operating characteristic (ROC) curve was plotted to compare a variable with their combinations to evaluate the effect of CTCs on improving the accuracy of the VPI prediction. The area under the curve (AUC) was calculated for each index. Statistical analyses were performed with SPSS v17.0 (SPSS Inc., Chicago, USA) and MedCalc v15.2 (MedCalc Software, Mariakerke, Belgium). All two-sided P values less than 0.05 denoted statistical significance.

\section{Results}

\section{Patient and tumor characteristics}

Three hundred and ninety-one patients with peripheral T1N0M0 adenocarcinoma (201 female and 190 male) were included in this study (Figure 2). Pleural tags or pleural contact was present for 234 patients, while the remaining 157 cases had no pleural involvement. One hundred and ninety-six patients with radiographic pleural involvement (196/234, 83.8\%) underwent lobectomy, which was statistically significantly more than those without involvement (113/157, 72.0\%). VPI was confirmed by elastic staining in 49 patients with radiographic pleural involvement (49/234, 20.9\%), consisting of 34 patients diagnosed as PL1 and 15 as PL2, while it was absent in those without pleural involvement. Significant differences were also found between patients with and without radiographic pleural involvement for tumor size, nodule type, tumor location, and predominant histological pattern, 


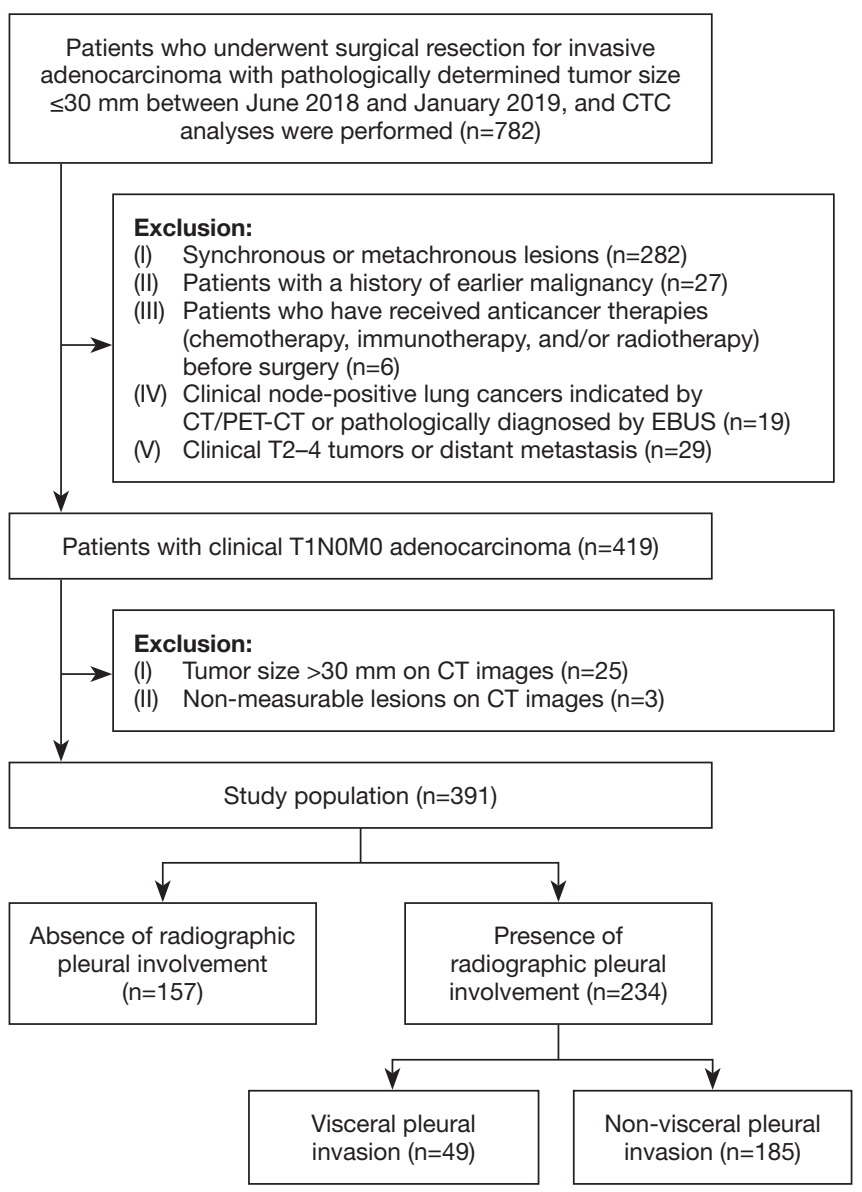

Figure 2 Flowchart of the study population. CT, computed tomography; CTC, circulating tumor cells; EBUS, endobronchial ultrasound; PET, positron emission tomography.

along with the elastic staining for VPI and type of resection (all $\mathrm{P}<0.05$ ). Detailed clinicopathological characteristics for the 391 patients are shown in Table 1.

\section{Analysis of the association between VPI and clinicopathologic characteristics for patients with radiographic pleural involvement}

For the 234 patients with CT features associated with VPI, various clinicopathological features were analyzed with relation to VPI, and the data are shown in Table 2. The median CTC level of $10.42 \mathrm{FU} / 3 \mathrm{~mL}$ of blood was applied to predict the presence of VPI in this cohort of 234 patients. Statistically significant differences between patients with and without VPI were found for preoperative level of CEA, CTC levels, tumor size, nodule type, and the predominant histological pattern (all $\mathrm{P}<0.05)$. At the same time, there were no significant differences between tumor location and lymph node metastasis (both $\mathrm{P}>0.05$ ). The univariate analysis also confirmed these findings. However, the multivariate logistic regression analysis only produced correlations between increased occurrence of VPI and tumor size [adjusted odds ratio $(\mathrm{OR})=1.96,95 \%$ confidence interval $(\mathrm{CI}): 1.02-3.76$, Ptrend $=0.044]$, nodule type (adjusted OR=9.62, 95\% CI: 3.98-23.26, Ptrend $<0.001$ ), and CTC levels (adjusted OR $=4.62,95 \%$ CI: $2.05-10.44, \mathrm{P}<0.001 ;$ Table 2$)$.

\section{CT features, CTC levels, and their association with presence of VPI}

Associations between radiographic parameters and CTC levels with the presence of VPI in groups of patients with pleural contact and tag are presented in Table 3. Of the 234 patients with peripheral adenocarcinoma and CT features 
Table 1 Characteristics of patients with $\leq 30 \mathrm{~mm}$ peripheral lung adenocarcinoma ( $\mathrm{n}=391)$

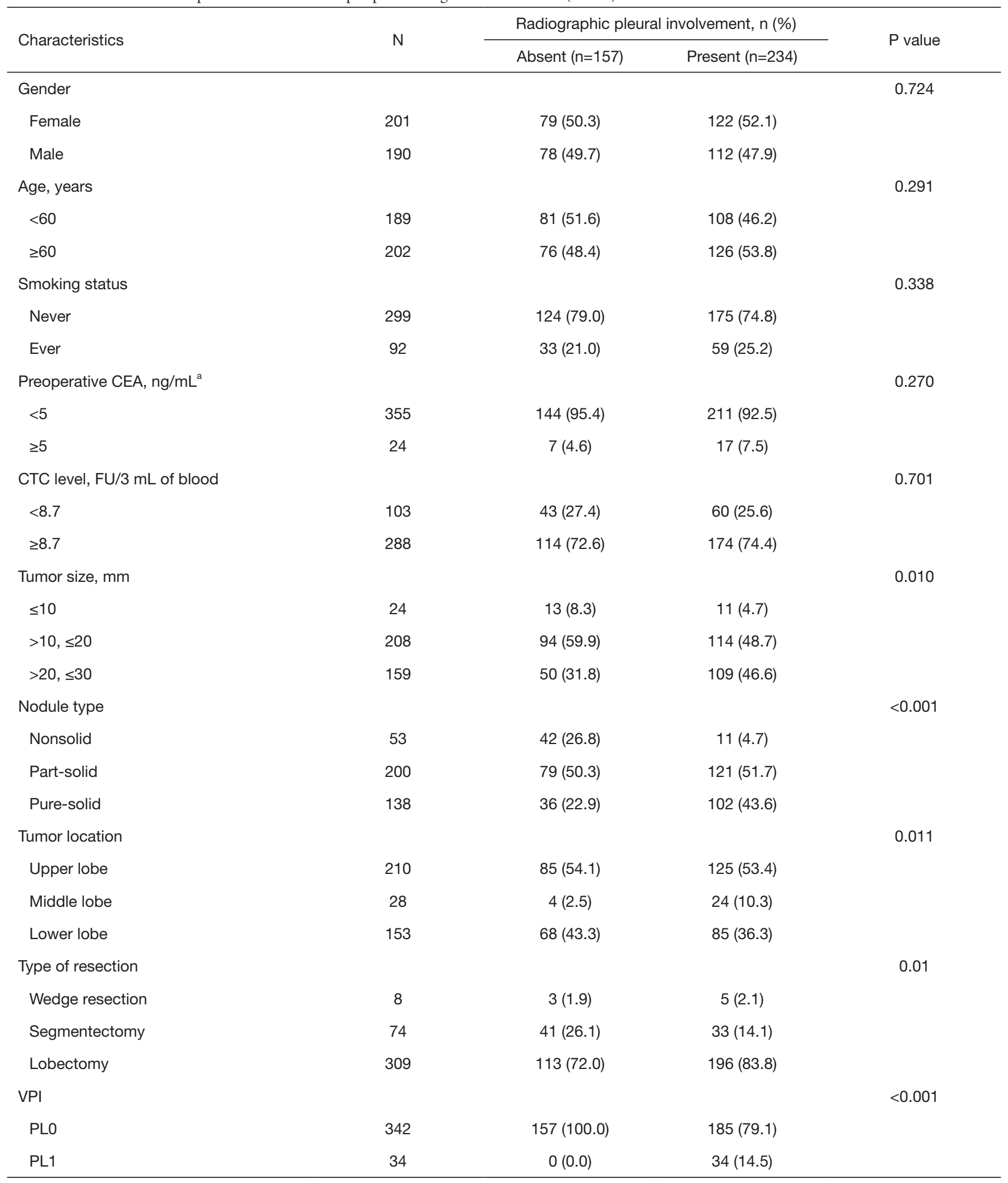

Table 1 (continued) 
Table 1 (continued)

\begin{tabular}{|c|c|c|c|c|}
\hline Characteristics & $\mathrm{N}$ & \multicolumn{2}{|c|}{ Radiographic pleural involvement, n (\%) } & $P$ value \\
\hline PL2 & 15 & $0(0.0)$ & $15(6.4)$ & \\
\hline pTNM stage & & & & 0.142 \\
\hline 1 & 362 & $149(94.9)$ & $213(91.1)$ & \\
\hline III & 15 & $6(3.8)$ & $9(3.8)$ & \\
\hline Lymph node metastasis & & & & 0.312 \\
\hline Negative & 365 & $149(94.9)$ & $216(92.3)$ & \\
\hline Positive & 26 & $8(5.1)$ & $18(7.7)$ & \\
\hline Acinar & 244 & $92(58.6)$ & $152(65.0)$ & \\
\hline Papillary & 32 & $15(9.6)$ & $17(7.3)$ & \\
\hline Micropapillary & 7 & $0(0.0)$ & $7(3.0)$ & \\
\hline Solid & 17 & $5(3.2)$ & $12(5.1)$ & \\
\hline Mucinous & 16 & $10(6.4)$ & $6(2.6)$ & \\
\hline
\end{tabular}

a, preoperative serum CEA values were available for 379 patients in our hospital. CEA, carcinoembryonic antigen; CTC, circulating tumor cells; FU, functional unit; VPI, visceral pleural invasion.

of pleural involvement associated with VPI, 141 had pleural tag and 93, pleural contact. VPI was more prevalent in tumors with pleural contact $(23 / 93,24.7 \%)$ compared to those with pleural tag $(26 / 141,18.4 \%)$. In the pleural contact group, significant differences were found between VPI-positive and VPI-negative groups for tumor size, solid portion size, ISP length, and CTC level, while such differences were only found for solid portion size and CTC level in the pleural tag group under univariate analysis. Multivariate logistic regression models further supported these findings, where the regression model showed that both for pleural contact and pleural tag groups, the CTC level was one of the independent indicators for the presence of VPI (adjusted OR $=4.12,95 \%$ CI: $1.09-15.66, \mathrm{P}=0.038$ for pleural contact; adjusted $\mathrm{OR}=5.34,95 \% \mathrm{CI}$ : $1.88-15.17$, $\mathrm{P}=0.002$ for pleural tag). Other independent indicators included an increase in ISP length (adjusted $\mathrm{OR}=1.48$, 95\% CI: $1.08-2.02, \mathrm{P}=0.014)$ for pleural contact, as well as solid portion size (adjusted OR $=1.14$, 95\% CI: 1.06-1.23, $\mathrm{P}<0.001$ ) for the pleural tag group.

\section{VPI can be predicted by bigh CTC levels along with radiographic features}

Next, we compared the ability of CTCs, with and without CT features, to identify VPI in peripheral adenocarcinomas. The analysis of the ROC curve compared the diagnostic performance of CTCs, CT features, including ISP length for the pleural contact and the size of the solid portion for the pleural tag, as well as the combined characteristics for both CTC and CT features (Figure 3). Under ROC curve analysis, the presence of both CT features and CTCs was more predictive for the presence of VPI, compared to CTCs or CT features alone (AUCs reaching statistical significance, $\mathrm{P}<0.05)$. Table 4 summarizes the resulting AUC and $95 \% \mathrm{CI}$ for the different markers, whereas the AUC of the CTC alone was 0.717 (95\% CI: 0.655-0.774). To further improve the diagnostic efficiency, CTC level and CT parameters were integrated, resulting in a model with significantly improved predictive efficiency for identifying VPI from tumors with CT-defined pleural involvement with both pleural contact and pleural tag subgroups (AUC 
Table 2 Analysis of the association between clinicopathological characteristics and visceral pleural invasion in patients with radiographic pleural involvement $(\mathrm{n}=234)$

\begin{tabular}{|c|c|c|c|c|c|c|c|c|}
\hline Characteristics & $\mathrm{N}$ & \multicolumn{3}{|c|}{ VPI, n (\%) } & \multicolumn{2}{|c|}{ Univariate analysis } & \multicolumn{2}{|c|}{ Multivariate analysis } \\
\hline Preoperative CEA, ng/mL ${ }^{a}$ & & & & 0.042 & & & & \\
\hline$<5$ & 211 & $170(94.4)$ & $41(85.4)$ & & Ref. & - & Ref. & - \\
\hline$\geq 5$ & 17 & $10(5.6)$ & $7(14.6)$ & & $2.90(1.04-8.08)$ & 0.041 & $1.13(0.33-3.92)$ & 0.845 \\
\hline$<10.42$ & 117 & $106(57.3)$ & $11(22.4)$ & & Ref. & - & Ref. & - \\
\hline$\geq 10.42$ & 117 & $79(42.7)$ & $38(77.6)$ & & $4.64(2.23-9.63)$ & $<0.001$ & $4.62(2.05-10.44)$ & $<0.001$ \\
\hline Tumor size, mm & & & & 0.039 & $1.84(1.03-3.28)$ & $0.040^{\mathrm{b}}$ & $1.96(1.02-3.76)$ & $0.044^{\mathrm{b}}$ \\
\hline$\leq 10$ & 11 & $10(5.4)$ & $1(2.0)$ & & & & & \\
\hline Nodule type & & & & $<0.001$ & $9.73(4.34-21.81)$ & $<0.001^{b}$ & $9.62(3.98-23.26)$ & $<0.001^{b}$ \\
\hline Nonsolid & 11 & $11(5.9)$ & $0(0.0)$ & & & & & \\
\hline Part-solid & 121 & $113(61.1)$ & $8(16.3)$ & & & & & \\
\hline Pure-solid & 102 & $61(33.0)$ & $41(83.7)$ & & & & & \\
\hline Tumor location & & & & 0.433 & $0.82(0.58-1.16)$ & $0.255^{b}$ & - & - \\
\hline Upper lobe & 125 & $96(51.9)$ & $29(59.2)$ & & & & & \\
\hline Middle lobe & 24 & $18(9.7)$ & $6(12.2)$ & & & & & \\
\hline Lower lobe & 85 & $71(38.4)$ & $14(28.6)$ & & & & & \\
\hline Acinar & 152 & $118(63.8)$ & $34(69.4)$ & & & & & \\
\hline Papillary & 17 & $12(6.5)$ & $5(10.2)$ & & & & & \\
\hline Micropapillary & 7 & $5(2.7)$ & $2(4.1)$ & & & & & \\
\hline Solid & 12 & $4(2.2)$ & 8 (16.3) & & & & & \\
\hline Mucinous & 6 & $6(3.2)$ & $0(0.0)$ & & & & & \\
\hline
\end{tabular}

${ }^{a}$, preoperative serum CEA values were available for 228 patients in our hospital; ${ }^{\mathrm{b}}$, $\mathrm{P}$ for trend. Cl, confidence interval; CEA, carcinoembryonic antigen; Ref., reference; CTC, circulating tumor cells; FU, functional unit; VPI, visceral pleural invasion. 
Table 3 Analysis of the association between radiographic features, CTC levels, and the presence of visceral pleural invasion in tumors with pleural contact or pleural tag $(\mathrm{n}=234)$

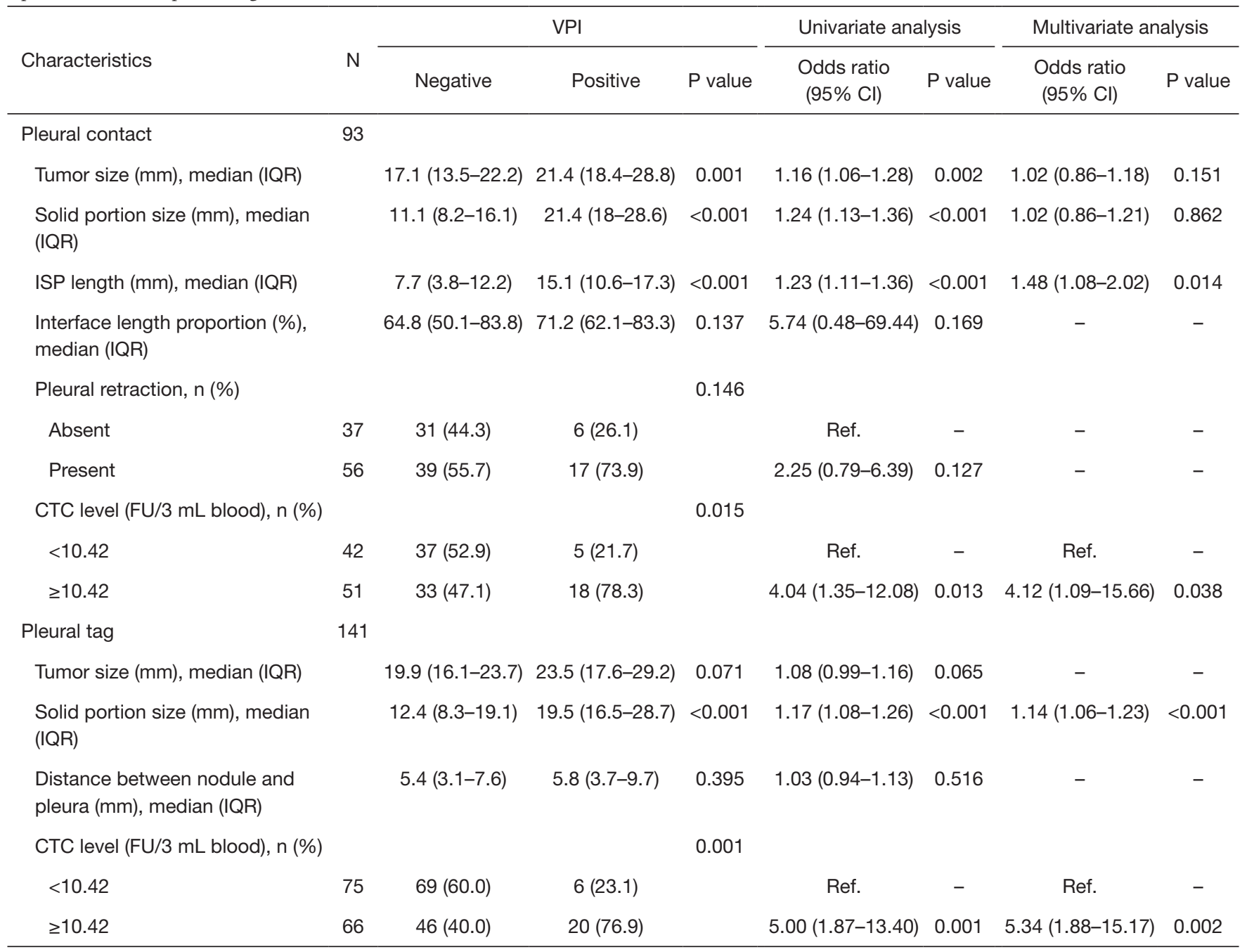

VPI, visceral pleural invasion; CI, confidence interval; IQR, interquartile range; ISP, interface solid portion; Ref., reference; CTC, circulating tumor cells; FU, functional unit.

$=0.921$ and 0.862 for pleural contact and pleural tag, respectively; both $\mathrm{P}<0.01$ ).

\section{Discussion}

Lung adenocarcinoma is the most common NSCLC type and is associated with a low 5 -year survival rate, ranging from $5-40 \%$, owing to $>75 \%$ of patients being diagnosed when it has progressed to its later stages (stages III-IV). By contrast, diagnosis at earlier stages, such as stage IA, yields a 5 -year survival rate of up to $60 \%$ (31). Therefore, multiple tests have been developed over the years to diagnose the cancer at earlier stages, such as chest CT scans, cytological analysis of respiratory fluids (ex. sputum, bronchoalveolar lavage), bronchoscopy, and lung tissue biopsies. However, all of those approaches have their limitations with respect to their accuracy and level of invasiveness. For instance, histological analyses of lung biopsies are considered the "gold standard" for accurately staging lung tumors, but they are often highly invasive and prone to complications from the biopsy procedure. Conversely, sputum cytology is noninvasive, only requiring a deep cough from a patient, but it is highly inaccurate for lung adenocarcinoma detection, and is unable to be used for staging $(32,33)$. As a result, noninvasive lung cancer staging has been a topic of considerable interest among radiologists and pulmonologists, as accurate 

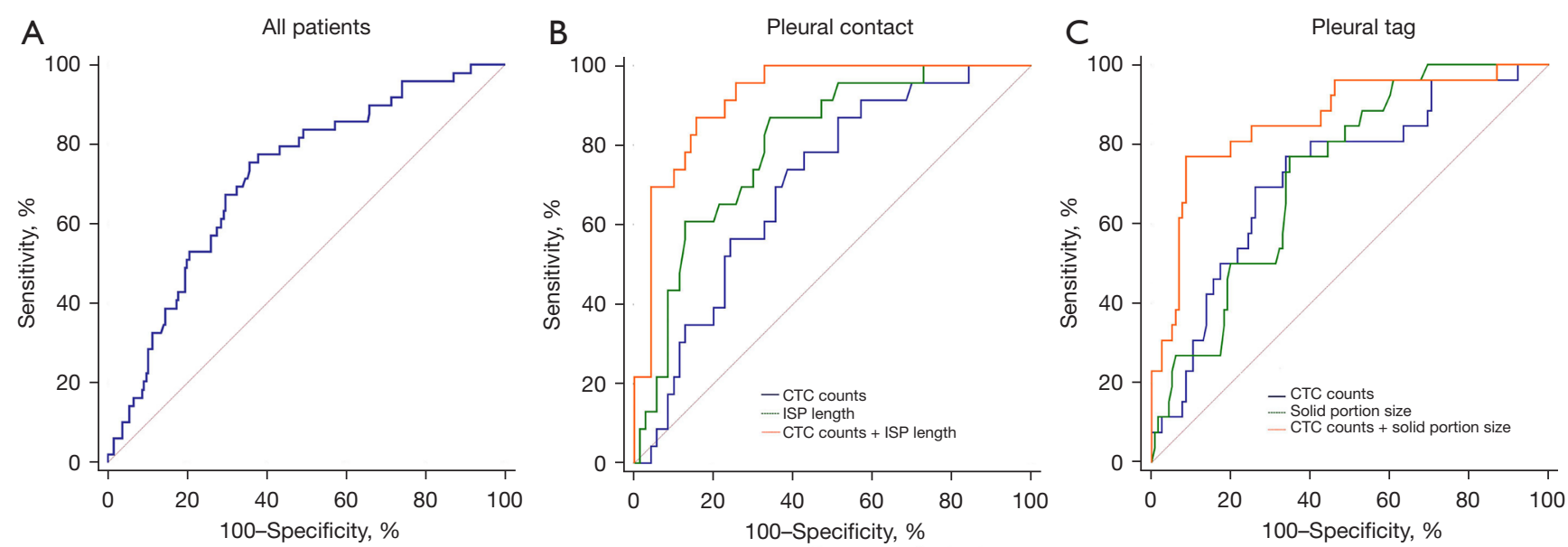

Figure 3 ROC curve analysis of CTCs combined with CT imaging features associated with VPI for all patients (A), patients with pleural contact (B), and patients with pleural tag (C). ROC, receiver operating characteristic; CTC, circulating tumor cells; CT, computed tomography; VPI, visceral pleural invasion; ISP, interface solid portion.

Table 4 Diagnostic efficacy of CTC levels and imaging features for predicting patients with visceral pleural invasion $(\mathrm{n}=234)$

\begin{tabular}{|c|c|c|c|c|c|c|}
\hline Group & AUC & $95 \% \mathrm{Cl}$ & $P$ value & Youden Index & Sensitivity (\%) & Specificity (\%) \\
\hline CTC counts & 0.717 & $0.655-0.774$ & - & 0.3983 & 75.51 & 64.32 \\
\hline \multicolumn{7}{|c|}{ Pleural contact, n=93 (VPI-positive vs. VPI-negative) } \\
\hline CTC counts & 0.702 & $0.599-0.793$ & 0.0003 & 0.3553 & 86.96 & 48.57 \\
\hline CTC counts + ISP length & 0.921 & $0.846-0.967$ & - & 0.7124 & 86.96 & 84.29 \\
\hline \multicolumn{7}{|c|}{ Pleural tag, n=141 (VPI-positive vs. VPI-negative) } \\
\hline CTC counts & 0.723 & $0.642-0.795$ & 0.0067 & 0.4314 & 69.23 & 73.91 \\
\hline Solid portion size & 0.730 & $0.648-0.801$ & 0.0051 & 0.4214 & 76.92 & 65.22 \\
\hline
\end{tabular}

AUC, area under the curve; $\mathrm{Cl}$, confidence interval; VPI, visceral pleural invasion; CTC, circulating tumor cells; ISP, interface solid portion.

staging is critical for further planning of appropriate therapeutic strategies. Among various characteristic malignant traits, researchers have extensively explored possibility of preoperative evaluation of VPI using various radiographic parameters (28). It has been documented in the literature that the CT features of VPI can be reliably used, with a fair degree of accuracy, ranging from $71-95 \%$, to detect its presence (34). This detection, in turn, aids to properly stage tumors preoperatively, in which tumors otherwise meeting the criteria for T1N0M0 classification would be re-staged as T2N0M0 in light of VPI presence, as recommended by the American Joint Committee on Cancer (AJCC) guidelines. Additionally, these CT features differ between solid and part-solid nodules, in which solid nodules with VPI display a larger contact length-to-size ratio, and multiple pleural tags, compared to VPI-negative nodules, while for part-solid nodules, VPI presence is associated with pleural contact and thickening for the solid component, as well as solid proportion $>50 \%$, nodule size $>2 \mathrm{~cm}$, and transgression/infiltration of the fissure (6,35). However, the wide range regarding the aforementioned accuracy for CT features has led to the formulation of various approaches, 
such as deep learning, to increase the diagnostic accuracy for VPI (29). Therefore, in this study, we developed an approach to improve VPI diagnostic accuracy by utilizing the presence of CTC counts for VPI detection to augment the accuracy of CT scans. Such an approach, to the best of our knowledge, has not been attempted in previous studies. We succeeded in filling this knowledge gap by demonstrating that it was possible to assess the potential presence of VPI using a combination of CT features and CTC levels. We found that there was a significant difference in CTC levels between VPI-positive and VPInegative peripheral adenocarcinomas, and that VPI presence can be predicted with high precision by combining CTC results with the identification of CT features, including ISP length for the pleural contact and the size of the solid portion for the pleural tag. In turn, the prediction of VPI aids in diagnosis and subsequent treatment of stage IA lung adenocarcinoma, as its presence necessitates the upgrading of a $\leq 3 \mathrm{~cm}$ tumor being staged as $\mathrm{T} 1$ to $\mathrm{T} 2$ under the current edition of AJCC staging classifications, owing to its association with worse prognoses. This upgrading, in turn, obliges the usage of more treatment options, such as lobectomy with systemic lymphadenectomy, to combat against the adenocarcinoma $(36,37)$.

VPI is a poor prognostic factor in NSCLC, possibly due to its association with skip N2 metastases (30). Lobectomy with lymph node dissection is the preferred standard treatment for NSCLC with VPI. However, sublobar resection has been shown to have comparable oncologic efficacy for well-selected patients with smallsized NSCLC (11). Hence, the preoperative diagnostic precision of VPI has a vital prognostic value in determining the needed extent of surgical intervention, i.e., lobectomy vs. sublobar resection. It has long been asserted that inadequate lymph node assessment, limited by sublobar resection, could result in a $39 \%$ higher risk of disease recurrence than lobectomy in early-stage NSCLC (38). In this study, pathologically confirmed VPI was never found in peripheral adenocarcinoma that manifested as completely nonsolid nodules $(0 \%)$; it was diagnosed in $8(6.6 \%)$ partsolid and $41(40.2 \%)$ pure-solid nodules, corresponding to findings from previous studies $(39,40)$. Several CT imaging characteristics are associated with VPI, including maximum tumor diameter, tumor-pleura interface length, and solid portion size $(6,41)$. Among these, Hattori et al. reported the solid component could potentially be a more potentially more significant predictive factor, compared to other CT imaging features, for malignant characteristics including elastic layer invasion and poorer clinical prognoses $(40,42)$. This observation is owed to the association of the solid component of lung adenocarcinoma nodules with the micropapillary/solid histopathological subtype, which have been shown to be correlated with decreased survivals, due to its higher invasiveness, compared to nodules with ground glass opacities (GGO) on CT scans. GGO, consisting of a hazy opacity that does not obscure underlying bronchial structures and pulmonary vessels in CT scans, conversely corresponds to the subsolid components of cancer nodules. These components, in turn, predominantly display a lepidic histological pattern, which has been observed in the literature to be less invasive than micropapillary/solid patterns, and thus associated with more favorable prognoses (43-45). These findings were supported in our study, in which the solid portion of the tumor, represented by ISP length of tumors with pleural contact, and the size of the solid portion for those with pleural tags, were found to be independent predictors of VPI based on CT features. VPI patients were also more frequently found to present with micropapillary and solid histologic subtypes associated with tumor invasion, which appeared as a solid component in CT scans. Furthermore, we found that the specificities reached $84.29 \%$ and $91.30 \%$ when assessed by a combination of the CT features of, respectively, ISP length and solid portion size, with CTC counts, therefore providing more accurate imaging features for VPI identification.

Liquid biopsy has been devised for lung cancer diagnosis, particularly for tumor locations where biopsies were usually difficult to perform. It has been extended to detect genomic alterations, monitor treatment responses, and evaluate non-invasive early-stage lung cancer (24). In recent years, there has been renewed interest, as clinicians and biologists become more attracted to the idea of CTC detection for NSCLC. However, standard protocols for CTC isolation and analysis for lung cancer have not been outlined, which is complicated by the non-uniformity of CTC surface antigens in their nature and abundance. This non-uniformity is reflective of their heterogeneity, which is owed to the cellular heterogeneity of their source tumors, in which some cells would be more "stem-like" in nature, and others "more differentiated". Such variation poses difficulties with a number of the current methods utilized to capture CTCs, such as immuno-magnetic binding, in that CTCs without specific surface markers of interest, such as EpCAM or CK, would not be detected. For instance, CTCs originating from NSLSC that are more "stem-like" in nature are less likely to express EpCAM, rendering immuno-magnetic 
binding for this surface marker as less effective, compared to capturing "more differentiated" CTCs (46). These changes in cell surface marker expression over time for CTCs, at different stages of differentiation, along with their rarity within the bloodstream, poses difficulties for their capture and enrichment, particularly when a specific cell expression profile is associated with a specific stage in cell development, such as the partial epithelial-to-mesenchymal transition phenotype with the early stages of metastasis, where being EpCAM- necessitates alternative identification approaches than immuno-magnetic binding (47). As a result, label-free approaches, combined with genetic sequencing methods, have been developed to overcome these limitations of marker-based capture, which, however, have been limited themselves by their low throughput. More recently, the limitations of label-free approaches, such as ISET, have been overcome via the development of highthroughput approaches, such as microfluidics involving the generation of Dean vortices to separate out the larger CTCs from the smaller blood cells, which, combined with single-cell genetic sequencing, have been able to obtain CTCs from the majority of tested patients, which otherwise would be missed from more traditional surface markerbased approaches, due to being mostly EpCAM- (46,48-50). This combination has also been found to be effective for other cancer types, such as breast and colorectal cancers $(47,51)$.

Isolation using FR was chosen as a preferable method in this study, due to the fact that it has low or no expression in normal lung tissues or macrophages, but is highly expressed on $\sim 80 \%$ of NSCLC cells (25). Its levels are higher in lung adenocarcinoma versus squamous cell carcinoma (52). The LT-PCR technique has been used to for detection of FRpositive CTCs, which yields high diagnostic accuracy (25). CTCs may enter the bloodstream due to tumor invasion through the elastic layer of blood vessels. The elastic layer consists of the layers of the lamina elastica externa (LEE) and the lamina elastica interna, with lymph and blood vessels in between (53). Hamasaki et al. found that tumor vascular involvement was significantly higher in patients with LEE involvement (54). Our study aimed to evaluate the associations between peripheral blood CTC counts and VPI based on these pathologic findings, and our results showed that 44 of 49 VPI-positive patients (89.8\%), and 130 of 185 VPI-negative patients (70.3\%), could be accurately diagnosed using the suggested threshold CTC value of $8.7 \mathrm{FU} / 3 \mathrm{~mL}$ in the serum. Our diagnostic rate was similar to other reported studies for VPI-negative lung adenocarcinoma in the literature $(26,55)$. It is also noted that a statistically significantly higher CTC level was found in VPI-positive lung adenocarcinoma cases. By itself, the CTC level detection had an AUC value of 0.717 , but higher values were obtained by adding CT features associated with pleural contact or tag. For example, increased values for sensitivity and specificity ( $76.92 \%$ and $91.30 \%$, respectively) were achieved for the pleural tag. Therefore, CTC counts can serve as a valuable supporting diagnostic biomarker for VPI prediction, supplementing the reported data that can be obtained from CT images (8). Such a combined approach could also be potentially applied to differentiate benign from malignant nodules. Other imaging approaches are also being used for VPI diagnoses, including magnetic resonance imaging, allowing for a potential wider application of CTC with advanced radiographic imaging in the future $(56,57)$.

There are some limitations in our study, one of which is potential selection bias resulting from a retrospective singlecenter study. In addition, all patients had invasive lung adenocarcinoma; adenocarcinoma in situ and minimally invasive adenocarcinoma were not included in this study as they could be accurately diagnosed using frozen tissue sections. Therefore, the choice of surgical procedure for those adenocarcinomas would not be affected, unlike with the presence of VPI for invasive adenocarcinoma, which would entail the usage of more aggressive treatment options in line with the requisite increase in staging from $\mathrm{T} 1$ to T2. Subsequent association analyses did not include tumors without pleural involvement, as they had no incidence of VPI by definition. The study also had a relatively small sample size, posing statistical challenges for exploring the potential differences between PL1 and PL2. Therefore, additional prospective studies, involving larger sample sizes and multiple institutes, are needed to validate these results in the future.

\section{Conclusions}

The present study demonstrates that a higher CTC level is an independent predictor for VPI in clinical T1N0M0 lung adenocarcinoma, along with ISP length for pleural contact and solid portion size for the pleural tag, with respect to VPI-associated CT features. The combination of both CTC level and these pleural-based CT features significantly improved the accuracy of predicting the incidence of VPI, compared to either of them alone. Thus, CTC analysis serves as a highly valuable ancillary approach, along with CT imaging features, for preoperative detection of VPI, greatly aiding in selecting effective surgical treatment 
approaches for invasive lung adenocarcinoma.

\section{Acknowledgments}

The authors appreciate the academic support from the AME Lung Cancer Collaborative Group.

Funding: This work was supported by the National Natural Science Foundation of China (NSFC) (No. 81802261).

\section{Footnote}

Reporting Checklist: The authors have completed the STARD reporting checklist. Available at https://dx.doi. org/10.21037/tlcr-21-896

Data Sharing Statement: Available at https://dx.doi. org/10.21037/tlcr-21-896

Conflicts of Interest: All authors have completed the ICMJE uniform disclosure form (available at https://dx.doi. org/10.21037/tlcr-21-896). TTS reports non-financial support from Novocure, Inc. and financial support from Galera Therapeutics, Inc., outside the submitted work. The other authors have no conflicts of interest to declare.

Ethical Statement: The authors are responsible for all aspects of the work and ensure that questions related to the accuracy or integrity of any part of the work are appropriately investigated and resolved. The study was approved by the Ethical Review Board of Shanghai Pulmonary Hospital (No. K21-012) and was conducted in accordance with the Declaration of Helsinki (as revised in 2013). Written informed consent was waived for this retrospective analysis.

Open Access Statement: This is an Open Access article distributed in accordance with the Creative Commons Attribution-NonCommercial-NoDerivs 4.0 International License (CC BY-NC-ND 4.0), which permits the noncommercial replication and distribution of the article with the strict proviso that no changes or edits are made and the original work is properly cited (including links to both the formal publication through the relevant DOI and the license). See: https://creativecommons.org/licenses/by-nc-nd/4.0/.

\section{References}

1. Siegel RL, Miller KD, Jemal A. Cancer statistics, 2019.
CA Cancer J Clin 2019;69:7-34.

2. Ijsseldijk MA, Shoni M, Siegert C, et al. Oncologic Outcomes of Surgery Versus SBRT for Non-Small-Cell Lung Carcinoma: A Systematic Review and Meta-analysis. Clin Lung Cancer 2021;22:e235-92.

3. Wo Y, Zhao Y, Qiu T, et al. Impact of visceral pleural invasion on the association of extent of lymphadenectomy and survival in stage I non-small cell lung cancer. Cancer Med 2019;8:669-78.

4. Rami-Porta R, Asamura H, Travis WD, et al. Lung cancer - major changes in the American Joint Committee on Cancer eighth edition cancer staging manual. CA Cancer J Clin 2017;67:138-55.

5. Detterbeck FC, Chansky K, Groome P, et al. The IASLC Lung Cancer Staging Project: Methodology and Validation Used in the Development of Proposals for Revision of the Stage Classification of NSCLC in the Forthcoming (Eighth) Edition of the TNM Classification of Lung Cancer. J Thorac Oncol 2016;11:1433-46.

6. Ahn SY, Park CM, Jeon YK, et al. Predictive CT Features of Visceral Pleural Invasion by T1-Sized Peripheral Pulmonary Adenocarcinomas Manifesting as Subsolid Nodules. AJR Am J Roentgenol 2017;209:561-6.

7. Kim H, Goo JM, Kim YT, et al. CT-defined Visceral Pleural Invasion in T1 Lung Adenocarcinoma: Lack of Relationship to Disease-Free Survival. Radiology 2019;292:741-9.

8. Hsu JS, Han IT, Tsai TH, et al. Pleural Tags on CT Scans to Predict Visceral Pleural Invasion of Non-Small Cell Lung Cancer That Does Not Abut the Pleura. Radiology 2016;279:590-6.

9. Sun W, Yang X, Liu Y, et al. Primary Tumor Location Is a Useful Predictor for Lymph Node Metastasis and Prognosis in Lung Adenocarcinoma. Clin Lung Cancer 2017;18:e49-55.

10. Moon Y, Choi SY, Moon MH. The prognosis of stage I non-small cell lung cancer with visceral pleural invasion and whole pleural adhesion after video-assisted thoracoscopic lobectomy: A single center retrospective study. J Thorac Dis 2020;12:5729-38.

11. Cao J, Yuan P, Wang Y, et al. Survival Rates After Lobectomy, Segmentectomy, and Wedge Resection for Non-Small Cell Lung Cancer. Ann Thorac Surg 2018;105:1483-91.

12. Kamigaichi A, Tsutani Y, Kagimoto A, et al. Comparing Segmentectomy and Lobectomy for Clinical Stage IA Solid-dominant Lung Cancer Measuring 2.1 to $3 \mathrm{~cm}$. Clin Lung Cancer 2020;21:e528-38. 
13. Yang X, Sun F, Chen L, et al. Prognostic value of visceral pleural invasion in non-small cell lung cancer: A propensity score matching study based on the SEER registry. J Surg Oncol 2017;116:398-406.

14. Liskova A, Samec M, Koklesova L, et al. Liquid Biopsy is Instrumental for 3PM Dimensional Solutions in Cancer Management. J Clin Med 2020;9:2749.

15. Chen X, Zhou F, Li X, et al. Folate receptor-positive circulating tumor cells as a predictive biomarker for the efficacy of first-line pemetrexed-based chemotherapy in patients with non-squamous non-small cell lung cancer. Ann Transl Med 2020;8:631.

16. Yin W, Zhu J, Ma B, et al. Overcoming Obstacles in Pathological Diagnosis of Pulmonary Nodules through Circulating Tumor Cell Enrichment. Small 2020;16:e2001695.

17. Papadaki MA, Messaritakis I, Fiste O, et al. Assessment of the Efficacy and Clinical Utility of Different Circulating Tumor Cell (CTC) Detection Assays in Patients with Chemotherapy-Naïve Advanced or Metastatic Non-Small Cell Lung Cancer (NSCLC). Int J Mol Sci 2021;22:925.

18. Chen L, Bode AM, Dong Z. Circulating Tumor Cells: Moving Biological Insights into Detection. Theranostics 2017;7:2606-19.

19. Plaks V, Koopman CD, Werb Z. Cancer. Circulating tumor cells. Science 2013;341:1186-8.

20. Castro-Giner F, Aceto N. Tracking cancer progression: from circulating tumor cells to metastasis. Genome Med 2020;12:31.

21. Manjunath Y, Upparahalli SV, Suvilesh KN, et al. Circulating tumor cell clusters are a potential biomarker for detection of non-small cell lung cancer. Lung Cancer 2019;134:147-50.

22. Aggarwal C, Wang X, Ranganathan A, et al. Circulating tumor cells as a predictive biomarker in patients with small cell lung cancer undergoing chemotherapy. Lung Cancer 2017;112:118-25.

23. Tartarone A, Rossi E, Lerose R, et al. Possible applications of circulating tumor cells in patients with non small cell lung cancer. Lung Cancer 2017;107:59-64.

24. Guibert N, Pradines A, Favre G, et al. Current and future applications of liquid biopsy in nonsmall cell lung cancer from early to advanced stages. Eur Respir Rev 2020;29:190052.

25. Chen X, Zhou F, Li X, et al. Folate Receptor-Positive Circulating Tumor Cell Detected by LT-PCR-Based Method as a Diagnostic Biomarker for Non-Small-Cell Lung Cancer. J Thorac Oncol 2015;10:1163-71.
26. Wang L, Wu C, Qiao L, et al. Clinical Significance of Folate Receptor-positive Circulating Tumor Cells Detected by Ligand-targeted Polymerase Chain Reaction in Lung Cancer. J Cancer 2017;8:104-10.

27. Detterbeck FC, Boffa DJ, Kim AW, et al. The Eighth Edition Lung Cancer Stage Classification. Chest 2017;151:193-203.

28. Nishino M. Using CT to Evaluate Visceral Pleural Invasion: Caution Is Advised. Radiology 2019;292:750-1.

29. Snoeckx A. Visceral pleural invasion: predictable on CT? Quant Imaging Med Surg 2019;9:2019-22.

30. Gorai A, Sakao Y, Kuroda H, et al. The clinicopathological features associated with skip N2 metastases in patients with clinical stage IA non-small-cell lung cancer. Eur J Cardiothorac Surg 2015;47:653-8.

31. Malapelle U, Vigliar E, Troncone G. Biomarkers predictive value in early stage non-small cell lung cancer. Transl Lung Cancer Res 2020;9:956-9.Malapelle U, Vigliar E, Troncone G. Biomarkers predictive value in early stage non-small cell lung cancer. Transl Lung Cancer Res 2020;9:956-9.

32. Nooreldeen R, Bach H. Current and Future Development in Lung Cancer Diagnosis. Int J Mol Sci 2021;22:8661.

33. Prabhakar B, Shende P, Augustine S. Current trends and emerging diagnostic techniques for lung cancer. Biomed Pharmacother 2018;106:1586-99.

34. Choi H, Kim H, Hong W, et al. Prediction of visceral pleural invasion in lung cancer on CT: deep learning model achieves a radiologist-level performance with adaptive sensitivity and specificity to clinical needs. Eur Radiol 2021;31:2866-76.

35. Heidinger BH, Schwarz-Nemec U, Anderson KR, et al. Visceral Pleural Invasion in Pulmonary Adenocarcinoma: Differences in CT Patterns between Solid and Subsolid Cancers. Radiol Cardiothorac Imaging 2019;1:e190071.

36. Chen T, Luo J, Wang R, et al. Visceral pleural invasion predict a poor survival among lung adenocarcinoma patients with tumor size $\leq 3 \mathrm{~cm}$. Oncotarget 2017;8:66576-83.

37. Zhang T, Zhang JT, Li WF, et al. Visceral pleural invasion in $\mathrm{T} 1$ tumors $(\leq 3 \mathrm{~cm})$, particularly $\mathrm{T} 1 \mathrm{a}$, in the eighth tumor-node-metastasis classification system for non-small cell lung cancer: a population-based study. J Thorac Dis 2019;11:2754-62.

38. Subramanian M, McMurry T, Meyers BF, et al. LongTerm Results for Clinical Stage IA Lung Cancer: Comparing Lobectomy and Sublobar Resection. Ann Thorac Surg 2018;106:375-81. 
39. Kim HJ, Cho JY, Lee YJ, et al. Clinical Significance of Pleural Attachment and Indentation of Subsolid Nodule Lung Cancer. Cancer Res Treat 2019;51:1540-8.

40. Hattori A, Suzuki K, Matsunaga T, et al. Visceral pleural invasion is not a significant prognostic factor in patients with a part-solid lung cancer. Ann Thorac Surg 2014;98:433-8.

41. Iizuka S, Kawase A, Oiwa H, et al. A risk scoring system for predicting visceral pleural invasion in non-small lung cancer patients. Gen Thorac Cardiovasc Surg 2019;67:876-9.

42. Hattori A, Matsunaga T, Takamochi K, et al. Prognostic impact of a ground glass opacity component in the clinical T classification of non-small cell lung cancer. J Thorac Cardiovasc Surg 2017;154:2102-2110.e1.

43. Moon Y, Sung SW, Lee KY, et al. The importance of the lepidic component as a prognostic factor in stage I pulmonary adenocarcinoma. World J Surg Oncol 2016; $14: 37$.

44. Lin G, Li H, Kuang J, et al. Acinar-Predominant Pattern Correlates With Poorer Prognosis in Invasive Mucinous Adenocarcinoma of the Lung. Am J Clin Pathol 2018;149:373-8.

45. Xi J, Yin J, Liang J, et al. Prognostic Impact of Radiological Consolidation Tumor Ratio in Clinical Stage IA Pulmonary Ground Glass Opacities. Front Oncol 2021;11:616149.

46. Zeinali M, Lee M, Nadhan A, et al. High-Throughput Label-Free Isolation of Heterogeneous Circulating Tumor Cells and CTC Clusters from Non-Small-Cell Lung Cancer Patients. Cancers (Basel) 2020;12:127.

47. Wang X, Deng L, Gjertsen BT. A microfluidic device for differential capture of heterogeneous rare tumor cells with epithelial and mesenchymal phenotypes. Anal Chim Acta 2020;1129:1-11.

48. Dai Z, Gu XY, Xiang SY, et al. Research and application

Cite this article as: Shi J, Li F, Yang F, Dong Z, Jiang Y, Nachira D, Chalubinska-Fendler J, Sio TT, Kawaguchi Y, Takizawa H, Song X, Hu Y, Duan L. The combination of computed tomography features and circulating tumor cells increases the surgical prediction of visceral pleural invasion in clinical T1N0M0 lung adenocarcinoma. Transl Lung Cancer Res 2021;10(11):4266-4280. doi: 10.21037/tlcr-21-896 of single-cell sequencing in tumor heterogeneity and drug resistance of circulating tumor cells. Biomark Res 2020;8:60.

49. Batth IS, Mitra A, Rood S, et al. CTC analysis: an update on technological progress. Transl Res 2019;212:14-25.

50. Lim SB, Lim CT, Lim WT. Single-Cell Analysis of Circulating Tumor Cells: Why Heterogeneity Matters. Cancers (Basel) 2019;11:1595.

51. Nanduri LK, Hissa B, Weitz J, et al. The prognostic role of circulating tumor cells in colorectal cancer. Expert Rev Anticancer Ther 2019;19:1077-88.

52. Nunez MI, Behrens C, Woods DM, et al. High expression of folate receptor alpha in lung cancer correlates with adenocarcinoma histology and EGFR corrected mutation. J Thorac Oncol 2012;7:833-40.

53. Warth A, Muley T, Herpel E, et al. A histochemical approach to the diagnosis of visceral pleural infiltration by non-small cell lung cancer. Pathol Oncol Res 2010;16:119-23.

54. Hamasaki M, Kato F, Koga K, et al. Invasion of the inner and outer layers of the visceral pleura in p'T1 size lung adenocarcinoma measuring $\leq 3 \mathrm{~cm}$ : correlation with malignant aggressiveness and prognosis. Virchows Arch 2012;461:513-9.

55. Yu Y, Chen Z, Dong J, et al. Folate receptor-positive circulating tumor cells as a novel diagnostic biomarker in non-small cell lung cancer. Transl Oncol 2013;6:697-702.

56. Zhang Y, Kwon W, Lee HY, et al. Imaging Assessment of Visceral Pleural Surface Invasion by Lung Cancer: Comparison of CT and Contrast-Enhanced Radial T1Weighted Gradient Echo 3-Tesla MRI. Korean J Radiol 2021;22:829-39.

57. Zhang W, Duan X, Zhang Z, et al. Combination of CT and telomerase+ circulating tumor cells improves diagnosis of small pulmonary nodules. JCI Insight 2021;6:148182. 